\title{
Image Segmentation using various Algorithms for Identifying Hotspot in Infrared Thermal Images of Weld Pool and for Estimation of Weld Bead Width
}

\author{
N. Chandrasekhar and M Vasudevan
}

\author{
Advanced Welding Process and Modeling Section \\ Materials Technology Division \\ Indira Gandhi Centre for Atomic Research, \\ Kalpakkam, Tamilnadu, India - 603102 \\ e-mail: dev@igcar.gov.in
}

\begin{abstract}
It is necessary to develop intelligent feedback control system to monitor and control weld bead geometry during TIG welding in real-time to facilitate remote repair welding in nuclear reactors. The experiments were carried out using IR camera on stainless steel plates and 30 samples were cut and bead width (BW) was measured. For identifying hot spot in the weld pool, Cellular Automata (CA) image segmentation algorithm is developed and BW was measured from the images. The performance is compared with K-Means and Fuzzy C-Means algorithms. The RMS error was found to be lowest for CA algorithm. Thus CA algorithm is proposed for IR thermal image processing.
\end{abstract}

\section{Introduction}

The TIG welding is widely employed for fabrication of structural components in the nuclear reactors. Most of the weld defects can be avoided during welding if correct weld bead geometry is ensured during welding. In order to ensure desired weld bead geometry, real time monitoring and control of weld bead geometry is essential during welding. The approach of monitoring, control and maintaining quality of weld bead is normally known as adaptive welding or intelligent welding. The intelligent welding as the name implies is that integrating welding machine, smart weld parameter sensing and control system using computational programming. In the part of developing intelligent welding system, sensing and control methodology process plays an important role. However, several sensing techniques have been adapted for monitoring and control of weld bead geometry which include acoustic sensor, weld pool oscillation sensor, optical sensor, ultrasonic sensor and vision sensor. Kovacevic et.al [1] is acquired IR thermal images with high shutter speed, image features were extracted and analysed with complicated image processing for oxidised weld pool surface area. The proposed image processing is to detect the weld pool boundary with accuracy of less than 100 ms. Hence, investigator proved that, the above methodology can overcome the influences caused by various disturbances during welding for processing thermal images. Ghanty et.al [2] have been used IR camera as a vision sensor, captured thermal images and developed a fuzzy rule based system for predicting weld bead geometry during TIG welding from the segmented thermal images. Vasudevan et.al $[3,4]$ have been proposed a methodology for estimating weld BW and DOP from the IR thermal images of weld pool and the identification of various weld defects from the recorded IR images. Chen and Chin [5] have employed IR thermography for adaptive control of DOP and BW during Gas Metal Arc welding and found linear relationship between full width half maximum and BW. One of the investigator has developed soft computing techniques using Artificial Neural Network and Adaptive Neuro Fuzzy Inference System for estimating weld BW and DOP from the IR thermal images of the weld pool [6]. Bowen Duan et.al [7] developed an effective segmentation approach based on cellular automata principle for blurry boundary, complicate structure and high speckle noise. The image features were extracted using energy function information of global image comparison and local image. The experiment results demonstrated to handle blurry boundaries in insensitive conditions. S. Wolframs [8] studied the fundamental behavior of $\mathrm{CA}$ and its local rule

The objective of the present work is for the comparison of performance of the various image segmentation algorithms such as KM, FCM with in-house developed CA algorithm for identifying hot spot region in the IR thermal image of the weld pool. From the segmented image, physical weld BW is computed and performance is compared. This technique will be to implemented by feed-back monitoring and control system in real-time during TIG welding of austenitic stainless steel. For this study, welding experiments were carried out to record IR thermal images of weld pool in real time at various TIG welding process parameters. The recorded IR thermal images were further processed using image processing algorithms for extracting features and for comparison of their performance by estimating weld BW.

\section{Experimental setup}

For acquiring IR thermal images in real time, JADE Cedip make IR camera is mounted at an angle of 45 degree on automatic TIG welding machine of high precision four axis computerized servo motor controlled TIG welding machine with high accurate water cooled torch Lincoln TIG 375A DC power source. The camera is positioned with welding torch to capture in real time IR thermal images of the weld pool for maintaining constant focal distance between weld pool and IR camera. The IR camera is calibrated for the temperature range of 473 to $1973^{\circ} \mathrm{K}$ with an accuracy of $\pm 1 \%$ over entire 
range. In order to avoid interference of arc radiation on the infra red intensity of the weld pool, a band pass filter of range 4.99 to $5.1 \mu \mathrm{m}$ was used to pass only the radiation from the weld pool to the camera. The experiments were conducted on squared butt joint of $6 \mathrm{~mm}$ thick 316LN SS plates of dimension $300 \mathrm{~mm}$ long $\times 250 \mathrm{~mm}$ using integrated automatic TIG welding machine setup with IR camera and shown in figure 1.

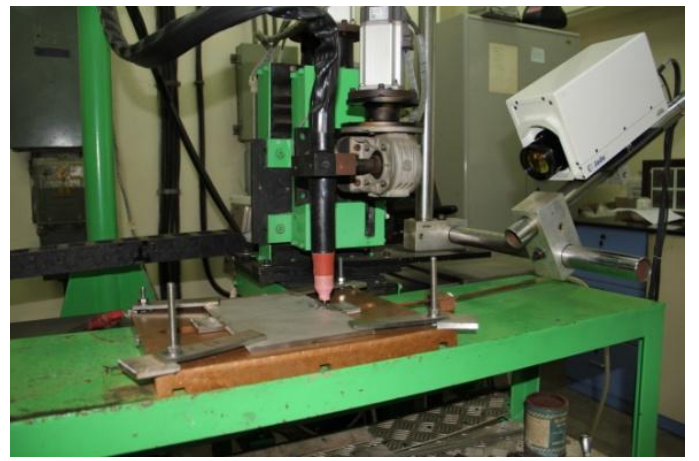

Fig. 1. Title TIG welding machine setup with IR camera

The welding was carried with variable currents of 150 to 175 Ampere in steps of 5 Ampere. 3 mm diameter of thoriated tungsten electrode was used and $1 \mathrm{~mm}$ of arc gap was set constant for all the experiments. The other welding parameters include torch speed of $2 \mathrm{~mm}$ per second, shielding gas flow rate of $10 \mathrm{Lpm}$ and arc gap of $1 \mathrm{~mm}$. After welding of 6 welded plates, 5 samples were cut on each plate with uniform distance. The cut samples were polished with automatic polishing machine and etched. Using high resolution of Nikon optical microscope, the BW was measured from the all 30 weld samples. The thermal images were captured and communicated through general purpose serial communication interface bus (GPIB) to Peripheral Component Interconnect $(\mathrm{PCl})$ of frame grabber. The image acquisition is facilitated by Altair software in the image acquisition and processing system. The stored thermal images in Altair work station is then processed and analyzed by Matlab software by industrial computer.

\section{Image segmentation and algorithm analysis}

The image segmentation involves identifying set of regions that are connected and overlapped which then acquires a unique region label that indicates the region it belong to. Nowadays, there are many methods are adopting such as morphology, statistical methods and partial differential equations. Although, there is still some disadvantages is in algorithms like suitability of certain images. In order to improve the segmentation accuracy CA method is adopted. However, concerning main issue the monitoring and control of BW in real-time during TIG welding process is the requirement for accurately identifying the hot spot from the IR thermal image of the weld pool using image processing algorithms. A CA based image processing algorithm has already been developed in house for image segmentation of IR thermal image of the weld pool [10]. However, the performance of the image processing algorithms in identifying the hot spot in the IR thermal image of the weld pool has not been evaluated so far. Therefore, the present work aims for comparing the performance of developed CA image segmentation algorithm with standard segmentation algorithms for identifying the hot spot in the IR thermal image of the weld pool. The performance of algorithm was compared in terms of extracted segment accuracy of the weld pool and execution time for different weld currents and identified key frames. Thus, the accuracy of the image segmentation for identifying the hot spot has been evaluated by comparing the estimated BW with that of the actually measured BW from the cut samples.

From the identified key frames, segment width is measured and correlated with the actual measured values of the weld bead geometry. Figure 2 shows the typical image of the weld pool captured by IR camera from identified key frame for image segmentation. The image pixel is calibrated in terms of $\mathrm{mm}$ using a measuring scale and value of 0.24 $\mathrm{mm}$ equaling one pixel has been determined. This calibrated measurement is used for segmenting accuracy by calculating the BW in mm corresponding to number of pixels is determined from the extracted hot spot region of the image segment. To calculate the execution time during implementation of algorithm, $3.2 \mathrm{GHz}$, processor of Intel Core i3 with 4 GB RAM on 64 bit Windows 7 operating system is used. The data were extracted for evaluating the image segment results such as RMS error and correlation coefficient between image segment BW and physically measured BW from the 30 identified key frames of different currents of IR thermal videos. The performance of comparison in terms of accuracy and execution time is shown in table 1. The algorithms were executed in Matlab platform for generating the number of clusters and segmentation analysis. In the following sub sections, implementation of algorithm, data extraction and result discussion for various image segmentation techniques is used in the present study is clearly described. 


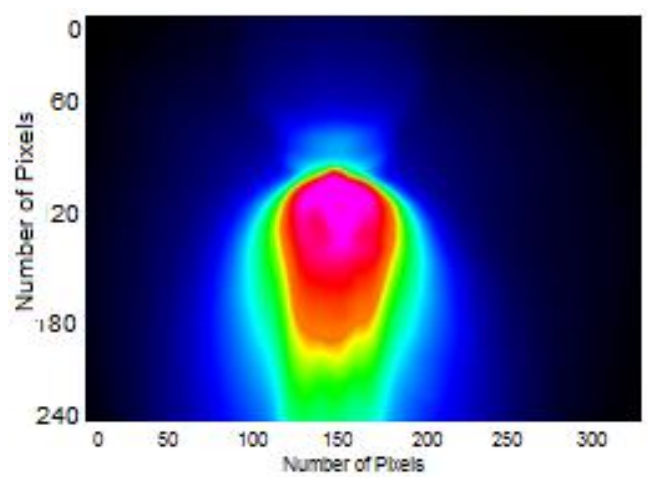

Fig. 2. Typical IR thermal image from identified key frame

\subsection{Cellular Automata image segmentation algorithm}

CA method is a natural choice for image processing because its local nature and parallel computing process. The concept was proposed by Ulam and Von Neumann and inspired by biological system. The important attributes of $\mathrm{CA}$ are dynamical system and consisting of a lattice of cell, each of which has number of associated discrete states. The states of these cells are being updated in the fashion of discrete time steps depending upon local state rules. Since space of CA is similar to the two dimensional of image matrix and the evolution of image has local transition rule. This transition rule determines state of the cell for further iteration, depending upon state of the cell itself and that of the other cells of its local neighborhood. The nearest adjacent cells is treated as local neighborhoods. CA is best tool for image processing which is easily to adopt parallel processing technique. Therefore, application of CA on image processing is still wide open. The main challenging in this algorithm is to determine the suitable transformation rule for required image segmentation purpose [9].

\subsubsection{Implementation of algorithm and data extraction}

To identify the hot spot region of the weld pool image, the sequence of algorithms follows the selection of input image from identified key frames of thermal video. Then the selected image frame is de-noised by average filter from field background noises for further processing. Null values of an empty row and column were padded around the filtered image to circulate the CA mapping matrix. The Moore's neighborhood is one of the commonly used neighborhood types in CA and shown in figure 3. The Moore's neighborhood consists of $3 \times 3$ matrix and it is configured as central cell (C) and neighborhood cells (c1-c8) which is around the central cell and shown in figure 4 . In order to apply transition rule, 6 number of $3 \times 3$ matrix is formed same as to Moore's neighborhood pattern. This matrix is mapped to 6 pairs of different combinations and shown in figure 4. Each cell represents the calibrated IR temperature magnitude of the image. These mapping matrixes are used to process the entire image matrix. The selection criterion of the central cell for segmentation is based on neighborhood states and transition rule. The considered transition rule is based on sum and difference between two sets (A1-A3, B1-B3) of cells. This determined value is again threshold to certain optimized value and iterate till to get the minimum error between predicted and actual values of the BW. The exact image segment width is computed by maximum number of pixels on the line scan of the image. Error is maintained always minimum by continuously varying threshold value with number of iterations. If central cell is met the above criteria then it is selected as weld pool hot spot region otherwise it is deselected. Figure 4 shows that various formats chosen for selection of central cell. The final segment is extracted when central cell converges with minimum errors.

The above followed steps is represented in flow chart and shown in figure 5. Figure 7 show that the extracted final segment for $150 \mathrm{~A}$ of $1563^{\text {th }}$ key frame for hot spot segmented region of the weld pool. The execution time varies from 310 to 500 milli seconds for different welding currents of identified key frames and it varies proportionately with number of iterations. The selected image segment width in $\mathrm{mm}$ is compared with physically measured BW in $\mathrm{mm}$. There is good agreement between the estimated BW and the actually measured BW. The RMS error value determined is 0.1083 and correlation coefficient is 0.9906 and plotted graph is shown in figure 6 . The execution time of algorithm is tabulated in table 1. 


\begin{tabular}{|c|c|c|}
\hline $\mathrm{c} 1$ & $\mathrm{c} 2$ & $\mathrm{c} 3$ \\
\hline $\mathrm{c} 8$ & $\mathrm{C}$ & $\mathrm{c} 4$ \\
\hline $\mathrm{c} 7$ & $\mathrm{c} 6$ & $\mathrm{c} 5$ \\
\hline
\end{tabular}

Fig. 3. Moore's neighborhood

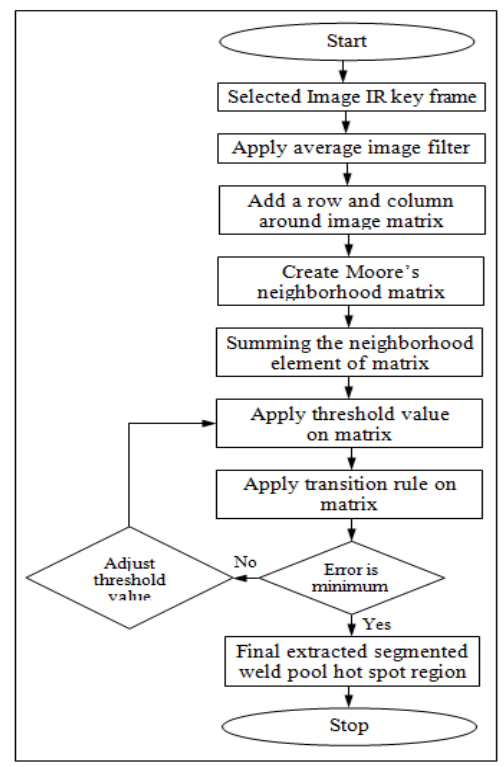

Fig. 5. Flowchart for $C A$ algorithm implementation

\begin{tabular}{|c|c|c|c|c|c|c|c|c|}
\hline Al & A2 & A3 & A2 & A3 & & $A_{2}$ & A3 & AA \\
\hline & P & & Al & P & B3 & Al & P & B4 \\
\hline B1 & B2 & B3 & & B1 & B2 & B1 & B2 & B3 \\
\hline A1 & & B1 & & Al & $A_{2}$ & B1 & A1 & $A_{2}$ \\
\hline $\mathrm{A} 2$ & $\mathbf{P}$ & B2 & B1 & $\mathbf{P}$ & A3 & B2 & $\mathbf{P}$ & $\mathrm{A}^{3}$ \\
\hline A3 & & B3 & B2 & B3 & & B3 & B4 & A4 \\
\hline
\end{tabular}

Fig. 4. CA mapping matrix

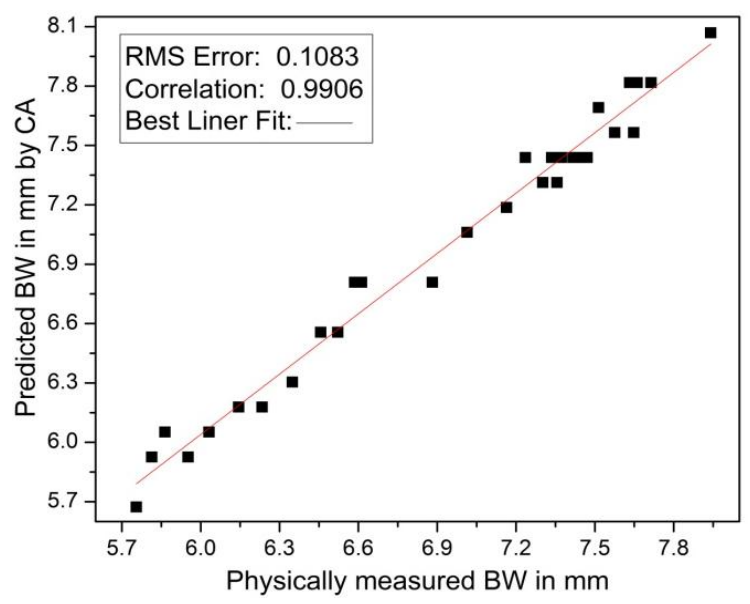

Fig. 6. Comparison of physically measured and predicted BW

Fig. 6. Compaison of physicaly measured and predicted BW

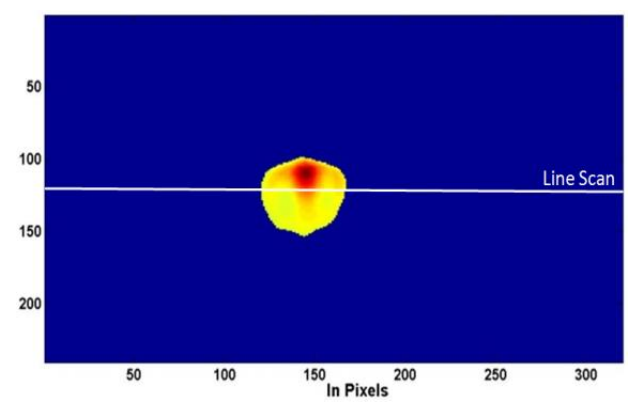

Fig.7. Typical segmented hot spot weld pool region

\subsection{K-Means image segmentation algorithm}

The K-Means (KM) algorithm is an iterative technique and is one of the widely used image segmentation algorithm. It is simple and easy to implement and has relatively low computational complexity. It is an unsupervised learning algorithm and solves the complex image segmentation issues. It is a least squares partitioning method that divide a collection of objects into $\mathrm{k}$ groups. The basic algorithm involves partitioning of an image into a certain number of $\mathrm{k}$ clusters. The aim is to define $\mathrm{k}$ centroids for each one cluster. These centroids are positioned in a random way and the best choice is to position them far away from each other as much as possible. Further step is to consider each point is related to given image matrix and associate it to the nearest centroid. After the assignment, the position of centres will be recalculated based on the distance measured and the algorithm has to repeat all the steps until convergence. 


\subsubsection{Implementation of algorithm and data extraction}

Figure 8 shows that the various stages involved for implementing KM algorithm on IR thermal images to extract hot spot region of weld pool. The algorithm is comprised of the following steps. The algorithm implementation starts with the input of selected IR thermal key image followed by applying de-noise technique of the input image through average filter. Then, the KM algorithm is applied on the filtered image to extract the hot spot region segment of the weld pool. From the extracted image segment, segment width is calculated by maximum number of pixels on $x$ axis of the image which is number of pixels along line scan as indicated on typical image and shown in figure. 10. The error value in mm is estimated by difference of BW measured by image segment and physically measured. If error is minimum then extracted segment is treated as hot spot segment region otherwise vary the number of clusters and apply the KM algorithm and calculated.

The figure 10 show that extracted final segment for $150 \mathrm{~A}$ of $1563^{\text {th }}$ key frame for hot spot region of the weld pool by KM algorithm. The number of clusters cluster varies from 10 to 15, iterations varies from 8 to 4 and execution time varies from 400 to $975 \mathrm{~ms}$. It is found that, execution time varies proportionately number of clusters and iterations for estimating bead geometry. The extracted image segment in $\mathrm{mm}$ is compared with physically measured BW in $\mathrm{mm}$. There is good agreement between the estimated and the actually measured BW. The RMS error value determined is 0.1172 and correlation coefficient is 0.9919 and plotted graph is shown in figure 9 . The execution time of algorithm is tabulated in table 1. It is observed that, comparison of performance with CA, KM algorithm produces less segment accuracy and slightly more execution time.

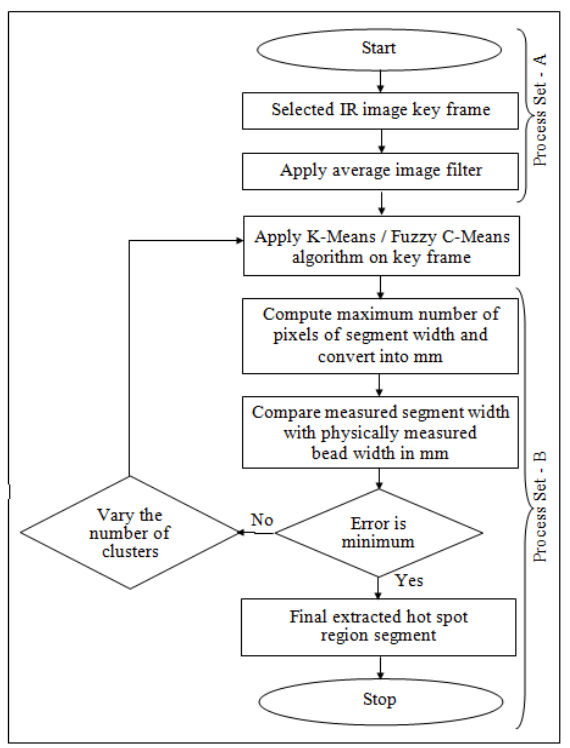

Fig. 8. Common flow chart for both K-Means and Fuzzy C-Means algorithm implementation

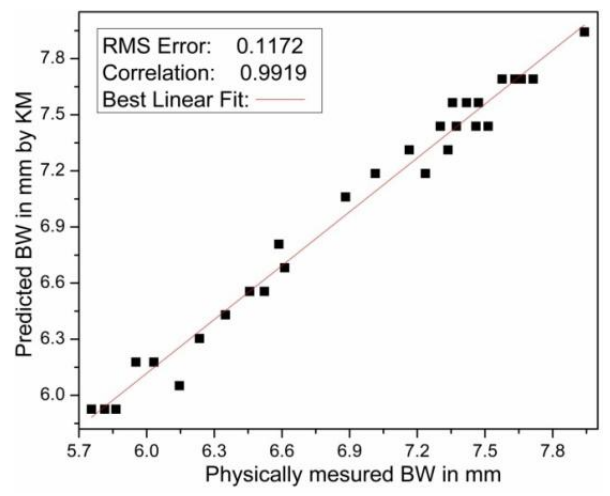

Fig. 9. Comparison of physically measured and predicted BW

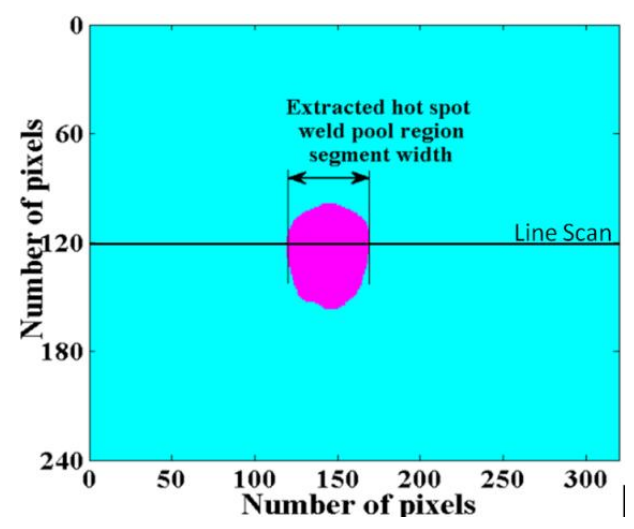

Fig. 10. Typical segmented hot spot weld pool region 


\subsection{Fuzzy C-Means image segmentation algorithm}

Fuzzy C-Means (FCM) is a method of clustering and it allows one pixel to belong to one or more clusters. It is a clustering technique which is separated from hard KM algorithm that employs hard partitioning. The FCM algorithm attempts to partition a finite collection of pixels into a collect of ' $C$ ' fuzzy clusters with respect to some given criteria. The FCM algorithm divides the images into number of homogeneous classes effectively. In terms of definition, an image $X=\left\{x_{i}\right\}, i €\{1,2 \ldots n\}$ is partially divided into $k$ number of clusters, where $x_{i}$ are the pixels of an image $X$ and $n$ is the total number of pixels. FCM clustering allows each pixel belong to all clusters. It is based on the minimization of the following objective function.

\subsubsection{Implementation of algorithm and data extraction}

The various stages involved for execution of algorithm is shown in figure 8 . The process set $A$ and $B$ which is indicated in figure 8 is similar to $\mathrm{KM}$ algorithm. By comparing with $\mathrm{KM}$ algorithm, it is noticed that the execution time is affecting due to generating number clusters and consuming more iterations. During the observation of process of algorithm, the FCM cluster varies from 15 to19, iterations varies from 10 to 15 and execution time varies from 750 to $1350 \mathrm{~ms}$. The figure 11 show that extracted final segment for $150 \mathrm{~A}$ of $1563^{\text {th }}$ key frame for hot spot region of the weld pool. The estimated hot spot segment width in $\mathrm{mm}$ is compared with the physically measure BW in mm as shown figure 12. There is good correlation between predicted and actual value of BW. The RMS error is calculated as 0.1527 and correlation coefficient is 0.9762 and shown in table 1 . The performance comparison with CA and KM it is observed that, segment accuracy is less and consumes execution time is slightly more because of generating more number of clusters.

The performance of the above algorithms in terms of the execution time and image segmentation accuracy is compared and shown in table 1. From the table, CA algorithm exhibit lowest execution time and minimum RMS error with good correlation coefficient values with other algorithms. Therefore, for the present case, CA is identified as the best algorithm for real time image processing of IR thermal image of the weld pool and integration with feed back control of BW during TIG welding.
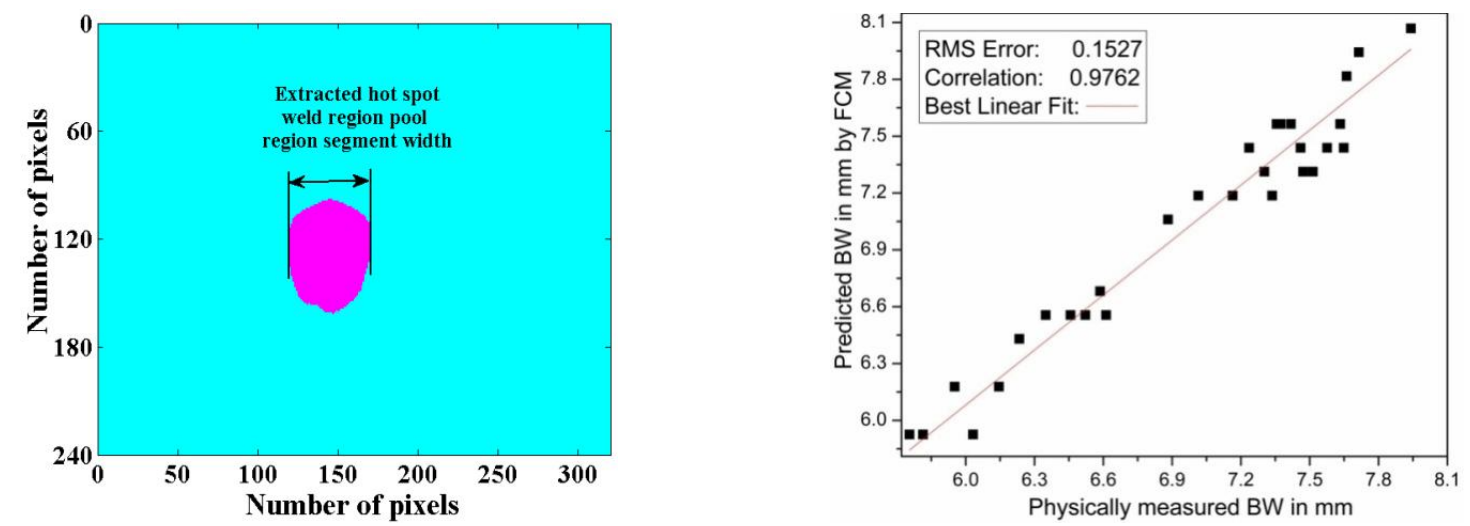

Fig. 11. Typical segmented hot spot weld pool region Fig. 12. Comparison of physically measured and predicted BW

Table. 1. Performance of comparison of various image segmentation algorithms in terms of accuracy.

\begin{tabular}{|l|l|l|l|}
\hline \multirow{2}{*}{$\begin{array}{l}\text { Segmentation } \\
\text { Algorithm }\end{array}$} & \multicolumn{2}{|c|}{ Accuracy } & \multirow{2}{*}{$\begin{array}{l}\text { Execution } \\
\text { Time (ms) }\end{array}$} \\
\cline { 2 - 4 } & RMS error & $\begin{array}{l}\text { Correlation } \\
\text { coefficient }\end{array}$ & $310-500$ \\
\hline CA & 0.1083 & 0.9906 & $400-975$ \\
\hline KM & 0.1172 & 0.9919 & $750-1350$ \\
\hline FCM & 0.1527 & 0.9762 & \\
\hline
\end{tabular}




\section{Conclusion}

A linear relation is found between the estimated weld bead width from the images and the actually measured bead width. The RMS error value of 0.1083 was obtained and CA algorithm is found to consumes less execution time. Hence, CA algorithm is found to be superior in terms of accuracy of estimation and faster execution time. Hence, CA image segmentation approach is found to be more suitable for hot spot identification from the IR thermal images of weld pool for estimating bead width during TIG welding in real-time.

\section{REFERENCES}

[1] Kovacevic R., Zhang Y.M., Ruan S., "Sensing and control of weld pool geometry for automate GTA welding", ASME Journal of Engineering for Industry, vol. 117(2), pp. 210-222, 1995.

[2] Ghanty P., Paul S., Roy A., Mukharjee D.P., Pal N.R., Vasudevan M., Kumar H., Bhaduri A.K., "Fuzzy rule based approach for predicting weld bead geometry in gas tungsten arc welding", Science and Technology of Welding and Joining, vol. 13(2), pp. 167-175(9), 2008.

[3] Vasudevan M., Chandrasekhar N., Maduraimuthu V., Bhaduri A.K., Raj B., "Real-time monitoring of weld pool during GTAW using infra-red thermography and analysis of infra-red thermal images", Welding in the World. vol. 55(7), pp. 83-89, 2010.

[4] Chokkalingham S., Chandrasekhar N., Vasudevan M., "Predicting depth of penetration and weld bead width from infra-red thermal image of weld pool using artificial neural network", Journal of Intelligent Manufacturing. vol. 23, pp.5, 2012.

[5] Chen W., Chin B. A., "Monitoring joint penetration using infrared sensing techniques", Welding Research Supplement. vol. 69(4), pp. 181s-185s, 1990.

[6] Subashini L., Vasudevan M., "Adaptive Neuro-Fuzzy Inference System (ANFIS) based model for predicting the weld bead width and depth of penetration from the infra-red thermal image of the weld pool", Metallurgical and Materials Transactions B, vol. 43(1), pp 145-154, 2012.

[7] Bowen Duan, Yan Liu, Xutang Zhang, and Hong Liu. "An effective image segmentation approach based on cellular automata principle." Industrial Engineering and Manufacturing Technology, Vol.5, 2015.

[8] Wolfram S., "Theory and applications of cellular automata", World Scientific, ISBN, 9971-50-123-6,1986,

[9] Aghajari E., Gharpure D. C., "Segmentation Evaluation of Salient Object Extraction Using K-Means and Fuzzy C-Means Clustering". International Journal of Advanced Electrical and Electronics Engineering (IJAEEE) ISSN (Print), vol. 1(2), 2278-8948, 2012.

[10] Chandrasekhar N., Vasudevan M., Bhaduri A. K., Jayakumar T., "Intelligent modeling for estimating weld bead width and depth of penetration from infra-red thermal images of the weld pool", Journal of Intelligent Manufacturing, doi: 10.1007/s10845-013-0762-x, vol. 26(1), pp. 59-71, 2013. 